\title{
Correction to: Anemia in patients with Covid-19: pathogenesis and clinical significance
}

\author{
Gaetano Bergamaschi ${ }^{1}\left[\right.$ [D $\cdot$ Federica Borrelli de Andreis ${ }^{1,2} \cdot$ Nicola Aronico $^{1} \cdot$ Marco Vincenzo Lenti $^{1,2}$. \\ Chiara Barteselli $i^{1,2}$. Stefania Merli ${ }^{1,2} \cdot$ Ivan Pellegrino ${ }^{1,2} \cdot$ Luigi Coppola $^{1,2}$. Elisa Maria Cremonte ${ }^{1,2}$. \\ Gabriele Croce $^{1,2}$. Francesco Mordà ${ }^{1,2}$. Francesco Lapia ${ }^{1,2}$. Sara Ferrari ${ }^{1,2}$ - Alessia Ballesio ${ }^{1,2}$. Alessandro Parodi ${ }^{1,2}$. \\ Francesca Calabretta ${ }^{1,2} \cdot$ Maria Giovanna Ferrari ${ }^{1,2}$. Federica Fumoso ${ }^{1,2}$. Antonella Gentile ${ }^{1,2} \cdot$ Federica Melazzini $^{1,2}$. \\ Antonio Di Sabatino ${ }^{1,2}$ on behalf of Internal Medicine Covid-19 Collaborators
}

Published online: 17 March 2021

(c) Springer Nature Switzerland AG 2021

\section{Correction to: Clinical and Experimental Medicine https://doi.org/10.1007/s10238-020-00679-4}

The original version of this article unfortunately contained a mistake. The correct information is given below.

The co-authorship of the members of "Internal Medicine Covid-19 Collaborators" was inadvertently not clearly indicated. The names of these members should have been clearly linked to the author line of the article, to ensure that the article can be found under each of their names in PubMed and other databases.

In order to rectify this situation, this erratum provides a corrected author line, found above, that includes the names of the members of "Internal Medicine Covid-19 Collaborators".

The given name and family name of the second author was incorrectly tagged in the $\mathrm{xml}$ data, therefore it is abbreviated wrongly as "de Andreis FB". The correct given name is Federica and family name is Borrelli de Andreis.

The original article has been corrected.

Acknowledgements Internal Medicine Covid-19 Collaborators: Giampiera Bertolino, Silvia Codega, Filippo Costanzo, Roberto Cresci, Giuseppe Derosa, Michele Di Stefano, Francesco Falaschi, Carmine Iadarola, Elisabetta Lovati, Pietro Carlo Lucotti, Alessandra Martignoni, Caterina Mengoli, Emanuela Miceli, Amedeo Mugellini,Chiara Muggia, Patrizia Noris, Elisabetta Pagani, Ilaria

The original article can be found online at https://doi.org/10.1007/ s10238-020-00679-4.

Gaetano Bergamaschi

n.bergamaschi@smatteo.pv.it

1 Department of Internal Medicine, San Matteo Hospital

Foundation, Piazzale Golgi, 27100 Pavia, Italy

2 University of Pavia School of Medicine, Pavia, Italy
Palumbo, Alessandro Pecci, Tiziano Perrone, Carla Pieresca, Paola Stefania Preti, Maria Concetta Russo, Carmelo Sgarlata, Luisa Siciliani, Andrea Staniscia, Francesca Torello Vjera, Giovanna Achilli, Andrea Agostinelli,Valentina Antoci, Francesco Banfi, Irene Benedetti, Michele Brattoli, Ginevra Cambiè, Roberta Canta, Sara Cococcia, Federico Conca, Mariangela Delliponti, Virginia Del Rio, Francesco Di Terlizzi, Anna Fiengo, Tommaso Forni, Giulia Freddi, Chiara Frigerio, Alessandra Fusco, Margherita Gabba, Matteo Garolfi, Giulia Gori, Giacomo Grandi, Paolo Grimaldi, Alice Lampugnani, Federica Lepore, Gianluca Lettieri, Jacopo Mambella, Chiara Mercanti, Alba Nardone, Luca Pace, Lucia Padovini, Lavinia Pitotti, Margherita Reduzzi, Giovanni Rigano, Giorgio Rotola, Umberto Sabatini, Lucia Salvi, Giovanni Santacroce, Jessica Savioli, Simone Soriano, Carmine Spataro, Debora Stefani.

The work was supported by Fondazione IRCCS Policlinico San Matteo, Pavia, Italy. The investigators are grateful to the patients who participated in this study and acknowledge the contribution of the healthcare professionals who faced the COVID-19 epidemic at the Internal Medicine Unit of San Matteo Hospital Foundation in Pavia, Italy.

Publisher's Note Springer Nature remains neutral with regard to jurisdictional claims in published maps and institutional affiliations. 